\title{
Spin and Magnetic Moment of ${ }^{33} \mathrm{Mg}$ : Evidence for a Negative-Parity Intruder Ground State
}

\author{
D. T. Yordanov, ${ }^{1}$ M. Kowalska, ${ }^{2,3}$ K. Blaum, ${ }^{2}$ M. De Rydt, ${ }^{1}$ K. T. Flanagan, ${ }^{1,3}$ P. Lievens, ${ }^{4}$ \\ R. Neugart, ${ }^{2}$ G. Neyens, ${ }^{1}$ and H. H. Stroke ${ }^{5}$ \\ ${ }^{1}$ Instituut voor Kern- en Stralingsfysica, Katholieke Universiteit Leuven, B-3001 Leuven, Belgium \\ ${ }^{2}$ Institut für Physik, Johannes Gutenberg-Universität Mainz, D-55099 Mainz, Germany \\ ${ }^{3}$ CERN, Physics Department, CH-1211 Geneva 23, Switzerland \\ ${ }^{4}$ Laboratorium voor Vaste-Stoffysica en Magnetisme, Katholieke Universiteit Leuven, B-3001 Leuven, Belgium \\ ${ }^{5}$ Department of Physics, New York University, New York, NY 10003, USA
}

(Received 29 July 2007; published 21 November 2007)

\begin{abstract}
We report on the first determination of the nuclear ground-state spin of ${ }^{33} \mathrm{Mg}, I=3 / 2$, and its magnetic moment, $\mu=-0.7456(5) \mu_{N}$, by combining laser spectroscopy with nuclear magnetic resonance techniques. These values are inconsistent with an earlier suggested 1 particle-1 hole configuration and provide evidence for a 2 particle- 2 hole intruder ground state with negative parity. The results are in agreement with an odd-neutron occupation of the 3/2 [321] Nilsson orbital at a large prolate deformation. The discussion emphasizes the need of further theoretical and experimental investigation of the island of inversion, a region previously thought to be well understood.
\end{abstract}

DOI: 10.1103/PhysRevLett.99.212501

Since introduced in nuclear physics, the concept of shell structure and magic numbers has governed our understanding of nuclear matter in atomic nuclei close to stability. With obtaining access to more exotic species, one could follow the nuclear-structure evolution towards extreme isospin values, in particular with increasing neutron excess in light systems. The onset of deformation in nuclei with a closed neutron $s d$ shell was discovered via the extra binding energy and anomalous spin of ${ }^{31} \mathrm{Na}[1,2]$, as well as the low-lying $2_{1}^{+}$state in ${ }^{32} \mathrm{Mg}$ [3]. Nuclear-reaction experiments directly revealed large prolate deformations in the three $N=20$ isotones ${ }^{30} \mathrm{Ne}[4],{ }^{31} \mathrm{Na}$ [5], and ${ }^{32} \mathrm{Mg}$ [6]. The observed phenomena are understood as an inversion of the normal spherical ground-state configurations, expected for these nuclei according to the traditional shell model, with deformed "intruder" states governed by particle-hole excitations over the $N=20$ shell gap. The transition to this "island of inversion" for $Z=12$ occurs at ${ }^{31} \mathrm{Mg}$, which has a nearly pure 2 particle-2 hole $(2 \mathrm{p}-2 \mathrm{~h})$ intruder ground state [7]. The next odd-mass isotope in the chain, ${ }^{33} \mathrm{Mg}$, is likely to be characterized by the same configuration. The nuclear moments of $\mathrm{Na}$ in the island of inversion [8,9] and studies of the neighboring even-even $\mathrm{Mg}$ isotopes [6,10] further provide evidence for $2 \mathrm{p}-2 \mathrm{~h}$ ground states. A $\beta$-decay study [11], on the other hand, tentatively assigns a $1 \mathrm{p}-1 \mathrm{~h}$ configuration to the ground state of ${ }^{33} \mathrm{Mg}$ with spin and parity $I^{\pi}=(3 / 2)^{+}$. An intermediate-energy Coulomb-excitation measurement [12] suggests a corrected spin-parity assignment $I^{\pi}=$ $(5 / 2)^{+}$(also 1p-1h) in accord with the evidence from a proton inelastic scattering study [13]. Thus, the groundstate properties of ${ }^{33} \mathrm{Mg}$ are a subject of debate, and a configuration that is at variance with the other isotopes in the region is proposed.

In this Letter, we report the direct measurement of the spin and magnetic moment of ${ }^{33} \mathrm{Mg}$, which allows us to
PACS numbers: 21.10.Hw, 21.10.Ky, 27.30.+t, 32.10.Fn

unambiguously characterize its ground-state configuration. This is of utmost importance for the theoretical modeling of the region, since the competition between $1 \mathrm{p}-1 \mathrm{~h}$ and $2 \mathrm{p}-2 \mathrm{~h}$ configurations is directly related to the size of the $N=20$ shell gap and the nucleon-nucleon interaction.

The neutron-rich ${ }^{31} \mathrm{Mg}$ and ${ }^{33} \mathrm{Mg}$ were produced at ISOLDE-CERN by $1.4-\mathrm{GeV}$ protons impinging on a thick uranium carbide target. Their short lifetimes enabled the use of the shortest cycle of $2 \times 10^{13}$ protons every $1.2 \mathrm{~s}$. Laser ionization was applied to select $\mathrm{Mg}$ [14] with an average yield for ${ }^{33} \mathrm{Mg}$ of $2.5 \times 10^{3}$ ions $/ \mu \mathrm{C}$. The radioactive beams were accelerated to $40 \mathrm{keV}$, mass analyzed, and delivered to the collinear laser spectroscopy setup [1517].

The hyperfine structure and nuclear gyromagnetic ratio are measured by employing laser spectroscopy and nuclear magnetic resonance techniques. The singly ionized magnesium (Mg II) resonantly interacts with circularly polarized UV laser radiation in the transition from the $3 s^{2} S_{1 / 2}$ ground state to the $3 p^{2} P_{3 / 2}$ excited state $\left(D_{2}\right.$ line at $279.635 \mathrm{~nm}$ [18]). Optical pumping takes place, polarizing the atomic angular momenta along the direction of the laser beam, parallel to a longitudinal weak magnetic field of about $0.6 \mathrm{mT}$. A strong magnetic field of about $0.3 \mathrm{~T}$, perpendicular to the propagation axis, rotates and decouples the electronic and nuclear angular momenta and leads to the final nuclear polarization. After implantation in a cubic crystal, the $\beta$-decay anisotropy of the polarized nuclear ensemble is observed with two telescopes of thin scintillators, placed at $0^{\circ}$ and $180^{\circ}$ with respect to the orientation axis. The experimental $\beta$ asymmetry $\left[N\left(0^{\circ}\right)-N\left(180^{\circ}\right)\right] /\left[N\left(0^{\circ}\right)+N\left(180^{\circ}\right)\right]$, constructed from the coincidence events, is measured as a function of the laser frequency in the reference frame of the atomic beam or as a function of a radio-frequency field applied to the crystal. 


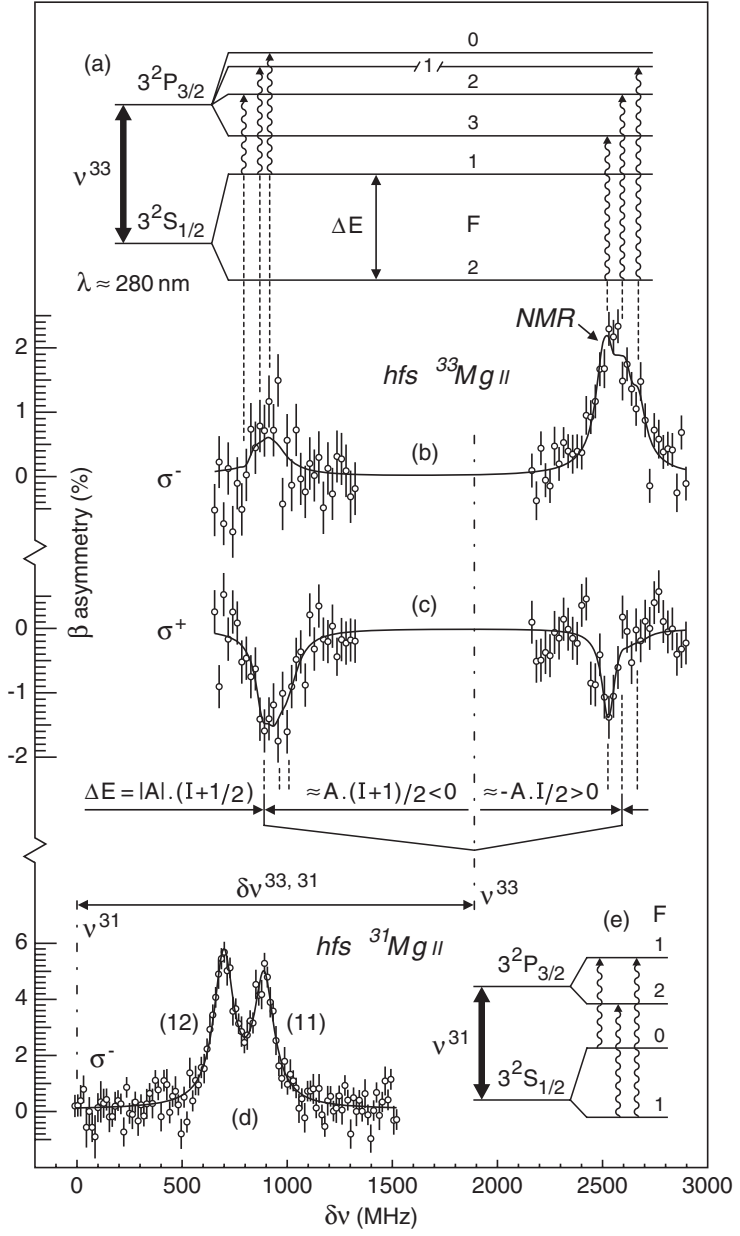

FIG. 1. (a) Hyperfine structure in the $D_{2}$ line of ${ }^{33} \mathrm{Mg}$ II $(I=$ $3 / 2, A<0$ ); (b), (c) $\beta$-asymmetry spectra for $\sigma^{-}$and $\sigma^{+}$optical pumping of ${ }^{33} \mathrm{Mg}$, implanted into $\mathrm{MgO}$; (d) $\beta$-asymmetry spectrum for $\sigma^{-}$optical pumping of ${ }^{31} \mathrm{Mg}(I=1 / 2, A<0)$, implanted into $\mathrm{MgO}$; (e) hfs scheme corresponding to (d). The transition $F=0 \rightarrow 1$ falls outside the frequency range of the figure.

The Doppler-shifted laser frequency is scanned over the hyperfine structure of the $D_{2}$ line, inducing transitions between the $3 s^{2} S_{1 / 2}$ and $3 p^{2} P_{3 / 2}$ hyperfine components, as shown in Figs. 1(a) and 1(e). The experimental spectra of ${ }^{33} \mathrm{Mg}$, obtained with $\sigma^{-}$and $\sigma^{+}$laser polarization, are displayed in Figs. 1(b) and 1(c). The frequency scale is given relative to the center of gravity of ${ }^{31} \mathrm{Mg}(I=1 / 2$ [7] ). The distance between the two groups of resonances in ${ }^{33} \mathrm{Mg}$ is determined by the splitting $\Delta E$ in the $3 s^{2} S_{1 / 2}$ ground state. This direct observable is related to the nuclear spin $I$ and the magnetic dipole hyperfine parameter $A$ by the equation: $\Delta E=|A|(I+1 / 2)$. The ratio $A / g$, where $g$ is the nuclear $g$ factor, is constant for an isotopic chain, if the hyperfine structure anomaly is neglected. This ratio is established for $\mathrm{Mg}$ II by the experimental work on the stable ${ }^{25} \mathrm{Mg}[19,20]$. Thus a $g$-factor measurement in
${ }^{33} \mathrm{Mg}$ leads to the determination of $A$, which in combination with the hfs splitting yields the nuclear ground-state spin.

Magnetic resonance measurements are carried out with the maximum polarization achieved for $\sigma^{-}$pumping in the higher frequency triplet of transitions [Fig. 1(b)]. The ions are implanted into the cubic crystal lattices of $\mathrm{MgO}$ (fcc) or $\mathrm{Pt}$ (ccp). A radio-frequency magnetic field of a few tenths of a $\mathrm{mT}$ is applied perpendicular to the static magnetic field $B$. In the vicinity of the Larmor frequency $\nu_{L}=g B \mu_{N} / h$ the experimental asymmetry is reduced by the resonant absorption of photons from the rf field. An example NMR spectrum of ${ }^{33} \mathrm{Mg}$ in $\mathrm{MgO}$, obtained with a small frequency modulation of $\pm 1 \mathrm{kHz}$, is presented in Fig. 2. Spectra of similar quality are obtained also in Pt. The reference isotope ${ }^{31} \mathrm{Mg}$ is implanted in both hosts in order to extract the ratio of the Larmor frequencies, which is independent of chemical and Knight shifts. The final result: $\nu\left({ }^{33} \mathrm{Mg}\right) / \nu\left({ }^{31} \mathrm{Mg}\right)=0.28130(16)$, combined with the $g$ factor of ${ }^{31} \mathrm{Mg}$ from Ref. [7], determines the absolute value of the ${ }^{33} \mathrm{Mg} g$ factor $|g|=0.4971(4)$. The error includes a systematic uncertainty of $10^{-4}$ due to a possible drift of the static magnetic field, estimated on the basis of precise measurements of the electric current flowing through the coils of the magnet. This value of the $g$ factor, corresponding to $\left|A_{g}\right|=866.2(7) \mathrm{MHz}$, in combination with the observed hfs splitting $\Delta E=1744(12) \mathrm{MHz}$ (Fig. 1) fixes the ground-state spin of ${ }^{33} \mathrm{Mg}$ to $I=3 / 2$.

The sign of the magnetic moment can be deduced from the data in two independent ways. The isotope shift between the stable ${ }^{24} \mathrm{Mg}$ and ${ }^{26} \mathrm{Mg}$ is measured to be $\delta \nu^{24,26}=3056(14) \mathrm{MHz}$, where the mass effect completely dominates. Hence, the isotope shift between ${ }^{31} \mathrm{Mg}$ and ${ }^{33} \mathrm{Mg}$ can be calculated to a good approximation from the relation: $\frac{\delta \nu^{31,33}}{\delta \nu^{24,26}}=\frac{m_{33}-m_{31}}{m_{31} m_{33}} \frac{m_{24} m_{26}}{m_{26}-m_{24}}$, where $m_{A}$ denotes the atomic masses. The result is $\delta \nu^{31,33}=1872(9) \mathrm{MHz}$, with statistical error and uncertainty due to the volume effect

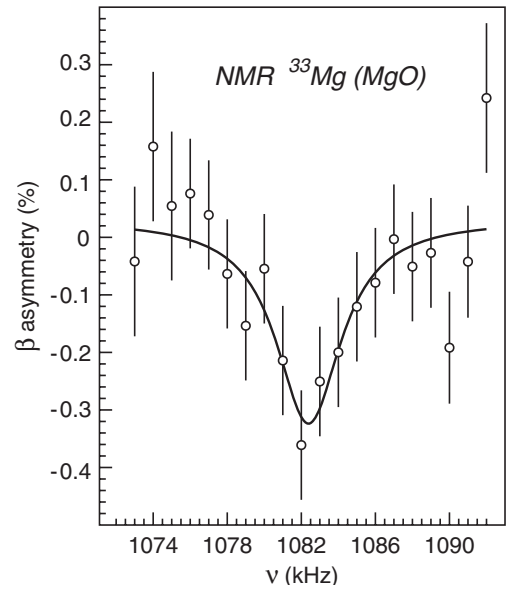

FIG. 2. NMR spectrum of ${ }^{33} \mathrm{Mg}$ implanted into $\mathrm{MgO}$, representing $34 \%$ of the total statistics. 
being insignificant for the considerations below. Based on the isotope shift one obtains the ${ }^{33} \mathrm{Mg}$ center of gravity, represented by the dash-dotted line $\nu^{33}$ in Fig. 1. The transitions starting from the higher angular momentum state $F=I+1 / 2(F=2$ for $I=3 / 2)$ are always closer to the center of gravity. In the case of ${ }^{33} \mathrm{Mg}$ their resonances appear at the higher frequency side of the spectra meaning that they start from the lower energy level. The inversion of the levels in $3 s^{2} S_{1 / 2}$ corresponds to a negative sign of the magnetic dipole hyperfine parameter. The same conclusion can independently be drawn from the simulated spectra for $\beta$-asymmetry detection of optical pumping $[15,16]$, represented by the fitted curves in Figs. 1(b) and 1(c). Since the ratio $A / g$ is positive for alkali-like atoms, the ground-state $g$ factor of ${ }^{33} \mathrm{Mg}$ is negative, resulting in a negative nuclear magnetic moment $\mu\left({ }^{33} \mathrm{Mg}\right)=g I \mu_{N}=$ $-0.7456(5) \mu_{N}$.

The negative sign of the magnetic moment is incompatible with the ground-state configuration of ${ }_{12}^{33} \mathrm{Mg}_{21}$ suggested in Ref. [11]. The proposed spin and parity $I^{\pi}=3 / 2^{+}$are associated with a $1 \mathrm{p}-1 \mathrm{~h}$ excitation over the $N=20$ shell gap. In the extreme single-particle shell-model picture, the properties of such a state are determined by an odd neutron in the $\nu 1 d_{3 / 2}$ orbital, which has a positive magnetic moment. The experimental negative sign of the magnetic moment can only be explained by an odd number of neutrons in the $p f$ shell, as these have negative Schmidt values for the orbitals $\nu 1 f_{7 / 2}$ and $\nu 2 p_{3 / 2}$. In this simplified manner one already arrives at the conclusion that the ground-state parity of ${ }^{33} \mathrm{Mg}$ is negative, determined by the negative parity of the latter two orbitals.

Large-scale shell-model calculations are carried out in the $s d$ - $p f$ model space with the code OXBASH [21], using the Hamiltonians from Refs. [22,23], designed specifically for the island of inversion. Mixing of states with a different number of particle-hole excitations $(\hbar \omega)$ is not taken into account. The neutron configuration space is restricted to $\nu\left(s d-1 f_{7 / 2}-2 p_{3 / 2}\right)$, as it is commonly undertaken in this region $[7,22,23]$. Two out of four valence protons are fixed on the $\pi 1 d_{5 / 2}$ orbital. The other two are confined within the $s d$ shell. Calculated nuclear moments for different particle-hole excitations $(0,1$, and $2 \hbar \omega)$ with spin $3 / 2$ are presented in Table I. Clearly, the experimental $g$ factor is only consistent with the $2 \mathrm{p}-2 \mathrm{~h}$ configuration and hence with a negative parity. This comparison further shows that

${ }^{33} \mathrm{Mg}$ has a nearly pure intruder ground state.

The charge-distribution deformation of ${ }^{33} \mathrm{Mg}$ has been determined to be $\beta_{C}=0.52$ (12) [12]. An axially symmetric rotor with a ground-state spin $I=K=3 / 2$ has a quadrupole moment directly related to this parameter [24]. The calculated value of $151(38) \mathrm{mb}$ is consistent with the quadrupole moment of the $2 \hbar \omega$ configuration in Table I. In a Nilsson-model picture the Fermi level for 21 neutrons coincides with an orbital having spin and parity
TABLE I. Calculated gyromagnetic ratios and quadrupole moments of different particle-hole excitations with $I=3 / 2$ in ${ }^{33} \mathrm{Mg}$, based on the interactions from Ref. [22], known as WBMB, and Ref. [23], which we refer to as SD-PF. Freenucleon $g$ factors and effective charges $e_{\pi}=1.5 e$ and $e_{\nu}=$ $0.5 e$ are used.

\begin{tabular}{|c|c|c|c|c|c|c|c|}
\hline$n(\hbar \omega)$ & $I^{\pi}$ & $g_{\text {free }}$ & $\begin{array}{l}\text { WBMB } \\
Q(\mathrm{mb})\end{array}$ & $E(\mathrm{keV})$ & $g_{\text {free }}$ & $\begin{array}{r}\text { SD-PF } \\
Q(\mathrm{mb})\end{array}$ & $E(\mathrm{keV})$ \\
\hline 0 & $3 / 2^{-}$ & -1.47 & -83 & 737 & -1.35 & -91 & 2374 \\
\hline 1 & $3 / 2^{+}$ & 0.75 & 135 & 399 & 0.78 & 140 & $283^{c}$ \\
\hline 2 & $3 / 2^{-}$ & $-0.45^{\mathrm{a}}$ & $147^{\mathrm{b}}$ & 1065 & $-0.47^{\mathrm{a}}$ & $157^{\mathrm{b}}$ & $0^{\mathrm{c}}$ \\
\hline
\end{tabular}

${ }^{\mathrm{a}}$ Experimental gyromagnetic ratio from this work $g=$ $-0.4971(4)$.

${ }^{\mathrm{b}} Q\left(\beta_{C}\right)=151(38) \mathrm{mb}$, calculated from $\beta_{C}$ [12].

${ }^{c}$ The $3 / 2^{+}(1 \hbar \omega)$ and $3 / 2^{-}(2 \hbar \omega)$ states are inverted in respect to the calculations presented in Ref. [11].

$I^{\pi}=3 / 2^{-}$only in section (a) of the $3 / 2$ [321] orbital, as shown in Fig. 3. This range is consistent with the matter deformation $\beta_{M}=0.47(8)$ from Ref. [13]. Correspondingly, the odd neutron in ${ }^{31} \mathrm{Mg}$ [7] occupies the $1 / 2$ [200] orbital in the segment (b). Similar deformation is detected in ${ }^{30} \mathrm{Ne}[4],{ }^{31} \mathrm{Na}$ [5], and ${ }^{32,34} \mathrm{Mg}[6,10]$. Hence, the present result associates ${ }^{33} \mathrm{Mg}$ with a prolate shape, which is common to all nuclei in the island of inversion.

A $\beta$-decay study of ${ }^{33} \mathrm{Mg}$ [25] reports no detectable feeding to the $5 / 2^{+}$states in ${ }^{33} \mathrm{Al}$, a fact that can now be understood, since these states are populated via firstforbidden $\beta$ transitions from the $3 / 2^{-}$ground state of ${ }^{33} \mathrm{Mg}$. All data available on the nuclear structure of ${ }^{33} \mathrm{Mg}$ are presented in a chronological order in Fig. 4. The large branching ratio observed in the $\beta$ decay of ${ }^{33} \mathrm{Na}$ [11] to the ground state of ${ }^{33} \mathrm{Mg}$ [Fig. 4(a)], which we deduced to have a negative parity, is difficult to understand, since ${ }^{33} \mathrm{Na}$ is expected to have a positive-parity ground state. The large

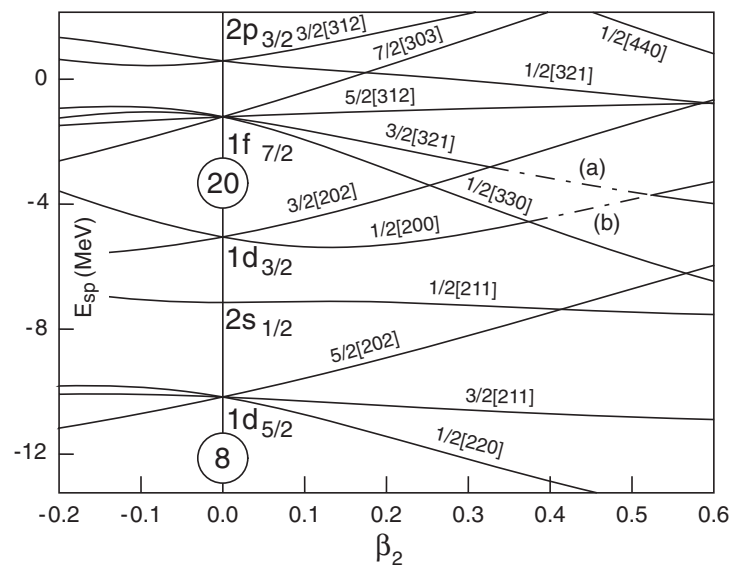

FIG. 3. Nilsson diagram in the $\nu\left(s d-1 f_{7 / 2}-2 p_{3 / 2}\right)$ configuration space calculated using universal Woods-Saxon potential [26,27]. (a), (b) Odd-neutron occupation in the ground states of ${ }^{33} \mathrm{Mg}$ and ${ }^{31} \mathrm{Mg}$, respectively. 


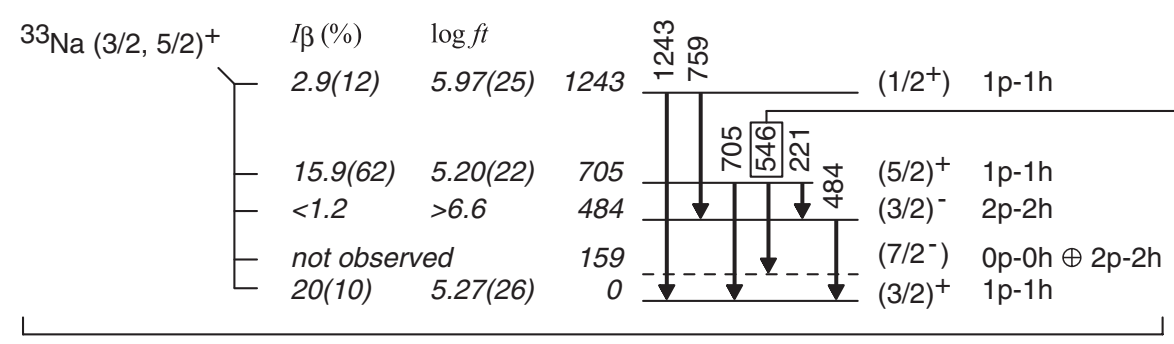

(a)

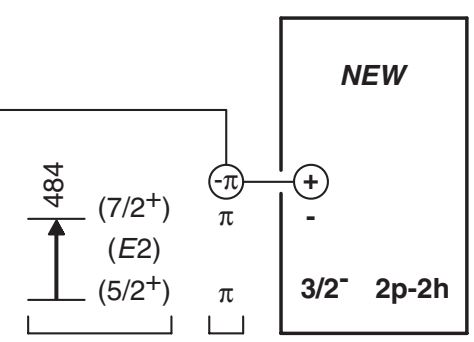

(b) (c)

(d)

FIG. 4. Nuclear-structure information on ${ }^{33} \mathrm{Mg}$ according to: (a) $\beta$ decay of ${ }^{33} \mathrm{Na}$ [11]; (b) intermediate-energy Coulomb excitation [12]; (c) proton inelastic scattering [13]; (d) this work.

cross section for Coulomb excitation to the $484 \mathrm{keV}$ level [12] has been tentatively associated with an $E 2$ transition [Fig. 4(b)]. Thus, a parity change from the ground state to this level, as suggested in Ref. [11], is unlikely. A proton inelastic scattering study [13] provides evidence supporting this assertion [Fig. 4(c)], which combined with the ground-state parity assignment from this work results in a negative parity for the $484 \mathrm{keV}$ level [Fig. 4(d)]. The latter experiment further associates the $546 \mathrm{keV}$ gamma with a parity opposite to the one of the ground state [Fig. 4(c)], since it has been observed in a neutron knockout reaction from ${ }^{34} \mathrm{Mg}$ and not in the proton scattering process. There is no experimental evidence that this decay originates from the $705 \mathrm{keV}$ level. Alternatively, the unobserved level in Fig. 4(a) should be replaced by an excited state at $546 \mathrm{keV}$, as pointed out in Ref. [11]. The positive parity associated with this transition according to our result [Fig. 4(d)] is an indication for a $1 \mathrm{p}-1 \mathrm{~h}$ configuration, thus showing a coexistence with $2 \mathrm{p}-2 \mathrm{~h}$ configurations. Clearly, these results are calling for new experiments to fully understand the structure of the excited states of ${ }^{33} \mathrm{Mg}$.

In conclusion, the ground-state spin and magnetic moment of a nucleus beyond $N=20$, in the middle of the "island of inversion", have been measured for the first time. The ${ }^{33} \mathrm{Mg}$ ground state is shown to have a nearly pure $2 \mathrm{p}-2 \mathrm{~h}$ intruder nature, contrary to the earlier suggested $1 \mathrm{p}-1 \mathrm{~h}$ configuration and in agreement with the structure of the other isotopes in this "island". The discussion demonstrates that the associated phenomena are not fully understood, emphasizing the necessity of further investigation.

We are grateful to B. A. Brown and A. Poves for sharing ideas. We acknowledge G. Rainovski for computing the Nilsson diagram. This work has been supported by the German Federal Ministry for Education and Research under Contracts No. 06MZ175 and No. 06MZ215, the FP6-EURONS Project No. RII3-CT-2004-506065, the IUAP Project No. P5/07 of OSCT Belgium and the
FWO-Vlaanderen. K. B. was supported by the Helmholtz Association for National Research Centers under Contract No. VH-NG-037. We would like to thank the ISOLDE technical group for their professional assistance.

[1] C. Thibault et al., Phys. Rev. C 12, 644 (1975).

[2] G. Huber et al., Phys. Rev. C 18, 2342 (1978).

[3] C. Détraz et al., Phys. Rev. C 19, 164 (1979).

[4] Y. Yanagisawa et al., Phys. Lett. B 566, 84 (2003).

[5] B. V. Pritychenko et al., Phys. Rev. C 63, 011305(R) (2000).

[6] T. Motobayashi et al., Phys. Lett. B 346, 9 (1995).

[7] G. Neyens et al., Phys. Rev. Lett. 94, 022501 (2005).

[8] M. Keim et al., AIP Conf. Proc. 455, 50 (1998).

[9] Y. Utsuno et al., Phys. Rev. C 70, 044307 (2004).

[10] H. Iwasaki et al., Phys. Lett. B 522, 227 (2001).

[11] S. Nummela et al., Phys. Rev. C 64, 054313 (2001).

[12] B. V. Pritychenko et al., Phys. Rev. C 65, 061304(R) (2002).

[13] Z. Elekes et al., Phys. Rev. C 73, 044314 (2006).

[14] U. Köster et al., Nucl. Instrum. Methods Phys. Res., Sect. B 204, 347 (2003).

[15] M. Keim et al., Eur. Phys. J. A 8, 31 (2000).

[16] M. Kowalska et al., Phys. Rev. C (to be published).

[17] W. Geithner et al., Hyperfine Interact. 129, 271 (2000).

[18] V. Kaufman and W. C. Martin, J. Phys. Chem. Ref. Data 20, 83 (1991).

[19] W. M. Itano and D. J. Wineland, Phys. Rev. A 24, 1364 (1981).

[20] F. Alder and F. C. Yu, Phys. Rev. 82, 105 (1951).

[21] B. A. Brown et al., MSU-NSCL Report No. 1289, 2004.

[22] E. K. Warburton et al., Phys. Rev. C 41, 1147 (1990).

[23] S. Nummela et al., Phys. Rev. C 63, 044316 (2001).

[24] A. Bohr and B.R. Mottelson, Nuclear Structure (Benjamin, New York, 1975), Vol. 2, p. 45-47.

[25] J. C. Angélique et al., AIP Conf. Proc. 831, 134 (2006).

[26] W. Nazarewicz et al., Nucl. Phys. A435, 397 (1985).

[27] S. Cwiok et al., Comput. Phys. Commun. 46, 379 (1987). 